\title{
Boundary Regularity for Some Nonlinear Elliptic Degenerate Equations ${ }^{\star}$
}

Haïm Brezis and Pierre-Louis Lions

Department of Mathematics, University of Paris VI, F-75230 Paris, France

Abstract. We consider the nonlinear elliptic degenerate equation

$$
-x^{2}\left(\frac{\partial^{2} u}{\partial x^{2}}+\frac{\partial^{2} u}{\partial y^{2}}\right)+2 u=f(u) \text { in } \Omega_{a},
$$

where

$\Omega_{a}=\left\{(x, y) \in \mathbb{R}^{2}, 0<x<a,|y|<a\right\}$

for some constant $a>0$ and $f$ is a $C^{\infty}$ functions on $\mathbb{R}$ such that $f(0)=f^{\prime}(0)=0$. Our main result asserts that: if $u \in C\left(\bar{\Omega}_{a}\right)$ satisfies

$$
u(0, y)=0 \text { for }|y|<a,
$$

then $x^{-2} u(x, y) \in C^{\infty}\left(\bar{\Omega}_{a / 2}\right)$ and in particular $u \in C^{\infty}\left(\bar{\Omega}_{a / 2}\right)$.

\section{Introduction}

This paper deals with the question of boundary regularity of solutions of a nonlinear elliptic degenerate equation of the form

$$
-x^{2} \Delta u+2 u=f(u) \text { in } \Omega_{a},
$$

where

$$
\begin{aligned}
\Delta & =D_{x}^{2}+D_{y}^{2} \\
\Omega_{a} & =\left\{(x, y) \in \mathbb{R}^{2} ; 0<x<a,|y|<a\right\}
\end{aligned}
$$

for some constant $a>0$, and $f$ is a $C^{\infty}$ function on $\mathbb{R}$ such that

$$
f(0)=f^{\prime}(0)=0 \text {. }
$$

* $\quad$ Sponsored by the United States Army under Contract No. DAAG 29-75-C-0024 
Our main result is the following:

Theorem 1. Assume $u \in C^{\infty}\left(\Omega_{a}\right) \cap C\left(\bar{\Omega}_{a}\right)$ satisfies (1) and

$$
u(0, y)=0 \text { for }|y|<a .
$$

Then $x^{-2} u(x, y) \in C^{\infty}\left(\bar{\Omega}_{a / 2}\right)$ and in particular $u \in C^{\infty}\left(\bar{\Omega}_{a / 2}\right)$.

Equation (1) occurs in the theory of multimeron solutions to Yang-Mills field equations [2]. More precisely the equation in [2] is :

$$
-x^{2} \Delta \psi+\psi^{3}-\psi=0 \text { in } \Omega_{a}
$$

together with the boundary conditions:

$$
\psi(0, y)= \pm 1
$$

If we set $u=\psi \mp 1$ we find

$$
-x^{2} \Delta u+(u \pm 1)^{3}-(u \pm 1)=0
$$

that is (1) with $f(u)=-u^{3} \mp 3 u^{2}$. In [3] it is only proved that $\psi$ is continuous up to the boundary (except at the points where $\psi$ changes sign). Theorem 1 shows that $\psi$ is $C^{\infty}$ up to the boundary (except at the points where $\psi$ changes sign).

\section{Some Lemmas}

The proof relies on some lemmas

Lemma 2. Assume $u \in C^{2}\left(\Omega_{a}\right) \cap C\left(\bar{\Omega}_{a}\right)$ satisfies :

$$
\left|-x^{2} \Delta u+2 u\right| \leqq \alpha\left(u^{2}+x^{4}\right) \text { on } \Omega_{a}
$$

for some constant $\alpha$, and

$$
u(0, y)=0 \text { for }|y|<a .
$$

Then, there is a constant $\beta$ such that

$$
|u(x, y)| \leqq \beta x^{2} \quad \text { on } \Omega_{a / 2} .
$$

Proof of Lemma 2. For $b<a$ set

$$
M_{b}=\operatorname{Sup}_{\Omega_{b}}|u| \text {. }
$$

Since by (5) $M_{b} \rightarrow 0$ as $b \rightarrow 0$,we may fix $b$ so small that

$$
\begin{gathered}
\alpha b^{2}<1 / 2 \\
\alpha M_{b}<1 / 400 .
\end{gathered}
$$

We shall establish that

$$
|u(x, 0)| \leqq A x^{2} \text { for } 0<x<b,
$$

where

$$
A=\operatorname{Max}\left\{\alpha b^{2}, \frac{100 M_{b}}{b^{2}}\right\} .
$$


The conclusion of Lemma 2 follows easily. In order to prove (8) we introduce the function

$$
v(x, y)=A x^{2}-B x^{4}+C y^{4},
$$

where $A$ is defined by (9),

$$
\begin{aligned}
& B=\frac{A}{2 b^{2}}, \\
& C=\frac{M_{b}}{b^{4}} .
\end{aligned}
$$

A direct computation shows that

$$
\begin{aligned}
-x^{2} \Delta v+2 v & \geqq \alpha\left(v^{2}+x^{4}\right) \quad \text { on } \Omega_{b}, \\
v(x, \pm b) & \geqq M_{b} \text { for } 0<x<b, \\
v(b, y) & \geqq M_{b} \text { for } 0<y<a, \\
\alpha \operatorname{Sup}_{\Omega_{b}} v \geqq 1, & \\
v & \geqq 0 \quad \text { on } \Omega_{b} .
\end{aligned}
$$

We now derive, using the maximum principle that

$$
u \leqq v \text { on } \Omega_{b} \text {. }
$$

Indeed by (14) and (15), $u \leqq v$ on $\partial \Omega_{b}$.

Suppose, by contradiction, that $(u-v)$ achieves a positive maximum at $\left(x_{0}, y_{0}\right) \in \Omega_{b}$. We would have

$$
\Delta(u-v)\left(x_{0}, y_{0}\right) \leqq 0 \text {. }
$$

On the other hand, we deduce from (4) and (13) that

$$
-x^{2} \Delta(u-v)+2 u-2 v \leqq \alpha\left(u^{2}-v^{2}\right) \text { on } \Omega_{b} .
$$

Therefore

$$
\begin{aligned}
2 & \leqq \alpha\left[u\left(x_{0}, y_{0}\right)+v\left(x_{0}, y_{0}\right)\right] . \\
& \leqq \alpha M_{b}+1 \quad[\text { by }(16)]
\end{aligned}
$$

and thus $\alpha M_{b} \geqq 1-$ a contradiction with (7).

Lemma 3. Under the assumptions of Theorem 1 there exist constant $\beta_{k}$ such that

$$
\left|D_{y}^{k} u(x, y)\right| \leqq \beta_{k} x^{2} \quad \text { on } \Omega_{a / 2},
$$

for all $k=0,1,2, \ldots$.

Proof of Lemma 3. Since $f(0)=0$ we have

$$
|f(u)| \leqq C|u| \quad \text { on } \Omega_{a}
$$


and by (1)

$$
|\Delta u| \leqq(C+2) \frac{|u|}{x^{2}} \text { on } \Omega_{a} .
$$

It follows from Lemma 2 that $\Delta u \in L^{\infty}\left(\Omega_{a / 2}\right)$. We deduce from the $L^{p}$ regularity theory (see e.g. [1]) that $u \in C^{1}\left(\bar{\Omega}_{a / 4}\right)$. In particular $D_{y} u \in C\left(\bar{\Omega}_{a / 4}\right)$ and

$$
D_{y} u(0, y)=0 \text { for }|y|<a / 4
$$

[since $u(0, y)=0$ for $|y|<a]$. Also, differentiating (1) with respect to $y$ we find

$$
-x^{2} \Delta\left(D_{y} u\right)+2\left(D_{y} u\right)=f^{\prime}(u) D_{y} u \quad \text { on } \Omega_{a} .
$$

By (2) we have

$$
\left|f^{\prime}(u)\right| \leqq C|u|
$$

and from Lemma 2 we see that

$$
\left|f^{\prime}(u)\right| \leqq C \beta x^{2}, \quad \text { on } \Omega_{a / 2} .
$$

\section{Consequently}

$$
\left|f^{\prime}(u) D_{y} u\right|^{2} \leqq C \beta\left(\left|D_{y} u\right|^{2}+x^{4}\right),
$$

and Lemma 2 applied to $D_{y} u$ shows that

$$
\left|D_{y} u\right| \leqq \beta_{1} x^{2} \quad \text { on } \Omega_{a / 8} .
$$

The conclusion of Lemma 3 for $k=1$ follows directly. When $k \geqq 2$ we proceed in a similar way, by induction, differentiating (1) $k$ times with respect to $y$.

Lemma 4. Assume $\varphi \in C^{2}(] 0, a[) \cap C([0, a])$ satisfies

$$
-x^{2} D_{x}^{2} \varphi(x)+2 \varphi(x)=h(x), \quad 0<x<a,
$$

where $h \in L^{\infty}(0, a)$.

Set $\psi(x)=x^{-2} \varphi(x)$, then

$$
D_{x} \psi(x)=-x^{-4} \int_{0}^{x} h(t) d t, \quad 0<x<a .
$$

Proof. Indeed we necessarily have

$$
\varphi(x)=\frac{C_{1}}{x}+C_{2} x^{2}+x^{2} \int_{x}^{a} \frac{d s}{s^{4}} \int_{0}^{s} h(t) d t
$$

for some constants $C_{1}$ and $C_{2}$. Since the last term remains bounded as $x \rightarrow 0$ we must take $C_{1}=0$, and the conclusion follows.

\section{Proof of Theorem 1}

We have by (1)

$$
-x^{2} D_{x}^{2} u+2 u=x^{2} D_{y}^{2} u+f(u) .
$$


Let $v(x, y)=x^{-2} u(x, y)$. We deduce from Lemma 4 that

$$
D_{x} v(x, y)=-x^{-4} \int_{0}^{x}\left[t^{2} D_{y}^{2} u(t, y)+f(u(t, y))\right] d t .
$$

Set $g(u)=u^{-2} f(u)$ so that by $(2), g$ is a $C^{\infty}$ function on $\mathbb{R}$. Changing the variable $t$ in (19) into $s=\frac{t}{x}$ we find

$$
D_{x} v(x, y)=-x \int_{0}^{1}\left[D_{y}^{2} v(s x, y)+v^{2}(s x, y) g\left(s^{2} x^{2} v(s x, y)\right)\right] s^{4} d s .
$$

It follows from Lemma 3 (applied with $k=0$ and $k=2$ ) that

$$
\left|D_{x} v(x, y)\right| \leqq C|x| \text { on } \Omega_{a / 2} .
$$

Next, if we differentiate (2) $k$ times with respect to $y$ we obtain, using Lemma 3, that

$$
\left|D_{x} D_{y}^{k} v(x, y)\right| \leqq C_{k} \quad \text { on } \Omega_{a / 2},
$$

for all $k$.

We may now differentiate (20) once with respect to $x$ and $k$ times with respect to $y$ and we find that

$$
\left|D_{x x} D_{y}^{k} v(x, y)\right| \leqq C_{k} \quad \text { on } \Omega_{a / 2}
$$

for all $k$. Proceeding by induction we obtain estimates for $D_{x}^{\ell} D_{y}^{k} v$ and the conclusion of Theorem 1 follows [note that we have even an estimate of the form $\left|D_{x}^{\ell} D_{y}^{k} v(x, y)\right| \leqq C x$ when $\ell$ is odd $]$.

Acknowledgements. We thank A. Jaffe for suggesting the problem and C. Goulaouic for useful discussions.

\section{References}

1. Agmon, S., Douglis, A., Nirenberg, L.: Estimates near the boundary for solutions of elliptic partial differential equations satisfying general boundary conditions. I. Comm. Pure Appl. Math. 12, 623-727 (1959)

2. Glimm, J., Jaffe, A.: Multiple meron solution of the classical Yang-Mills equation. Phys. Lett. B 73, 167-170 (1978)

3. Jonsson, T., McBryan, O., Zirilli, F., Hubbard, J. : An existence theorem for multimeron solutions to classical Yang-Mills field equations. Commun. Math. Phys. 68, 259-273 (1979)

Communicated by A. Jaffe

Received August 8, 1979 
